\title{
盐酸溶液和水热脱铝 HEU-1 分子篮的表征及其催化裂解性能
}

\author{
刘晓玲 ${ }^{1}$, 王 艳 ${ }^{1}$, 王旭金 ${ }^{1}$, 张亚飞 ${ }^{1}$, 巩赝军 ${ }^{1, *}$, 徐庆虎 ${ }^{1,2}$, 徐 君 ${ }^{3}$, 邓 风 ${ }^{3}$, 荬 涛 $^{1}$ \\ ${ }^{1}$ 中国石油大学 (北京)重质油国家重点实验室, CNPC 催化重点实验室, 北京 102249 \\ 2 中海油新能源投资有限责任公司，北京 100016 \\ 3 中国科学院武汉物理与数学研究所, 湖北武汉 430071
}

摘要: 采用 $\mathrm{HCl}$ 溶液和/或水热处理 $\mathrm{HEU}-1$ 分子篎, 利用 $\mathrm{X}$ 射线衍射、 $\mathrm{N}_{2}$ 吸附-脱附、 $\mathrm{X}$ 射线荧光光谱、 $\mathrm{NH}_{3}$ 程序升温脱附、 吡啶吸附傅里叶变换红外光谱和铝固体核磁共振等对脱铝处理的 HEU-1 分子篮进行了表征, 并考察了脱铝 HEU-1 分子篮上 正已烷的催化裂解性能. 结果表明, 采用 $\mathrm{HCl}$ 溶液处理 HEU-1 分子篮, 能有效脱除非骨架铝, 可调变样品的弱酸中心; 而水热 处理 HEU-1 分子篮, 能有效脱除骨架铝, 可调变样品的强酸中心. 水热处理和 $\mathrm{HCl}$ 溶液处理有效结合可以达到同时调变 HEU-1 分子篎的酸类型和酸密度的目的. 脱铝 HEU-1 分子篮上正己烷催化裂解反应结果表明, 氢转移反应被明显抑制, 丙烯 选择性提高. $600{ }^{\circ} \mathrm{C}$ 水热处理后再用 $\mathrm{HCl}$ 溶液处理的 $\mathrm{HEU}-1$ 分子篮于 $625^{\circ} \mathrm{C}$ 反应时丙烯选择性由未改性的 $21.9 \%$ 提高到 $35.2 \%$, 活性稳定性也由不足 $10 \mathrm{~h}$ 提高到 $40 \mathrm{~h}$.

关键词: HEU-1 分子筛; 脱铝; 水热处理；盐酸溶液处理；催化裂解；丙烯

中图分类号: O643 文献标识码: A

收稿日期: 2012-07-20. 接受日期: 2012-08-21.

*通讯联系人. 电话: (010)89733066; 传真: (010)89744979; 电子信箱: gongyj@cup.edu.cn

基金来源: 国家重点基础研究发展计划 (973 计划, 2010DFB40440); 国家自然科学基金 $(21176255,10979076$ ); 国际科技与交 流专项项目 (2012CB215002,); 中石油科技创新基金 (2010D-5006-0404).

本文的英文电子版(国际版)由 Elsevier 出版社在 ScienceDirect 上出版(http://www.sciencedirect.com/science/journal/18722067).

\section{Characterization and Catalytic Performance in $\boldsymbol{n}$-Hexane Cracking of HEU-1 Zeolites Dealuminated Using Hydrochloric Acid and Hydrothermal Treatments}

\author{
LIU Xiaoling ${ }^{1}$, WANG Yan ${ }^{1}$, WANG Xujin ${ }^{1}$, ZHANG Yafei ${ }^{1}$, GONG Yanjun ${ }^{1, *}$, XU Qinghu ${ }^{2}$, \\ XU Jun ${ }^{3}$, DENG Feng ${ }^{3}$, DOU Tao ${ }^{1}$ \\ ${ }^{1}$ State Key Laboratory of Heavy Oil Processing, CNPC Key Laboratory of Catalysis, China University of Petroleum-Beijing, \\ Beijing 102249, China \\ ${ }^{2}$ China National Offshore Oil Corporation New Energy Investment Co., Ltd., Beijing 100016, China \\ ${ }^{3}$ Wuhan Institute of Physics and Mathematics, Chinese Academy of Sciences, Wuhan 430071, Hubei, China
}

\begin{abstract}
A series of modified HEU-1 zeolites were prepared using hydrochloric acid treatment, hydrothermal treatment, and a combination of these. The parent and modified HEU-1 zeolites were characterized by X-ray diffraction, $\mathrm{N}_{2}$ adsorption-desorption isotherms, X-ray fluorescence, temperature-programmed desorption of ammonia, Fourier-transform infrared spectroscopy of adsorbed pyridine, and ${ }^{27} \mathrm{Al}$ solid-state magic-angle spinning nuclear magnetic resonance techniques. The catalytic cracking performances of the modified HEU-1 zeolites were evaluated on a fixed-bed microreactor at $625^{\circ} \mathrm{C}$, atmospheric pressure, and an $n$-hexane weight hourly space velocity of $2.0 \mathrm{~h}^{-1}$. The results showed that extra-framework alumina could be removed by directly treating with hydrochloric acid, and framework alumina could be removed by hydrothermal treatment. Acidity characterization showed that the weak acid sites of HEU-1 zeolites could be adjusted using acid treatment, and the number of strong acid sites could be reduced using hydrothermal treatment. The framework $n_{\mathrm{Si}}: n_{\mathrm{Al}}$ ratio was improved by using a combination of hydrothermal treatment and acid leaching. The acidities of the HEU-1 zeolites were adjusted and both the mesopore volume and external surface area were enhanced. In $n$-hexane cracking reactions, the dealuminated HEU-1 zeolite obtained by hydrothermal treatment for $4 \mathrm{~h}$ at $600{ }^{\circ} \mathrm{C}$ and then washing with hydrochloric acid exhibited the highest propylene production as a result of suppression of the hydrogen transfer reaction. At $625^{\circ} \mathrm{C}$, the selectivity for propylene on HEU-1(HT4-HCl) increased to $35.2 \%$ from $21.9 \%$,
\end{abstract}


and the lifetime of HEU-1(HT4-HCl) was also prolonged from not more than 10 to $40 \mathrm{~h}$.

Key words: HEU-1 zeolite; dealumination; hydrothermal treatment; hydrochloric acid treatment; catalytic cracking; propylene

Received 20 July 2012. Accepted 21 August 2012

*Corresponding author.Tel: +86-10-89733066; Fax: +86-10-89734979; E-mail: gongyj@cup.edu.cn

This work was supported by the National Basic Research Program of China (973 Program, 2012CB215002), the National Natural Science Foundation of China (10979076, 21176255), International Science and Technology Cooperation and Exchange program of China (2010DFB40440), and CNPC Innovation Foundation (2010D-5006-0404).

English edition available online at Elsevier ScienceDirect (http://www.sciencedirect.com/science/journal/18722067).

EU-1 分子篮是 1981 年由 Casci 等开发的一种 硅铝分子篮, 其 (001) 晶面具有一维十元环孔道, 且 在十元环主孔道上连接十二元环的侧袋 ${ }^{[1,2]}$. 研究表 明 ${ }^{[3,4]}$, 采用 $N, N, N, N^{\prime}, N^{\prime}, N^{\prime}$-六甲基-1,6 已二铵 (HM) 为模板剂合成的 EU-1 分子篮骨架中的 $\mathrm{Al}$ 原子定 向分布在十二元环的孔口和底部, 因而, 具有十二元 环的孔口和底部的两个活性中心. EU-1 特殊的侧 袋结构使其在 C8 芳烃的异构化反应中表现出良好 的活性和选择性.

法国国家石油研究院把 EU-1 分子篮引入二甲 苯异构化的催化剂中, 在相同的条件下表现出更高 的活性和选择性 ${ }^{[5,6]}$. Teketel 等 ${ }^{[7]}$ 研究了 EU-1 分子 篮在甲醇制烃类反应中的催化性能, 结果表明, EU-1 分子篮具有高的活性, 但氢转移活性也很高, 导致产物中烯烃的含量较低, 而芳烃的含量较高. Abrevaya 等 ${ }^{[8]}$ 考察了硅铝比为 29 35 的 EU-1 分子 篮上石脑油催化裂解反应性能, 发现 EU-1 分子篮 具有高的催化裂解活性, 但酸中心较多, 氢转移反应 严重, 使得低碳烯烃的选择性较低, 乙烯和丙烯选择 性之和仅有 $40 \%$, 产物中丙烷与丙烯的选择性相差 不大.

抑制烷烃裂解过程中的氢转移反应是增加低碳 烯烃选择性的关键, 催化剂的酸密度越高, 氢转移反 应的活性越高 ${ }^{[9 \sim 14]}$. 因此, 降低催化剂的酸密度是提 高反应低碳烯烃选择性的重要方法之一 ${ }^{[15 ~ 17]}$. 作为 有效地降低分子篮酸密度的方法, 酸处理和水热处 理广受关注 ${ }^{[15,16,18 \sim 24]}$. 水热处理能有效脱除分子篮

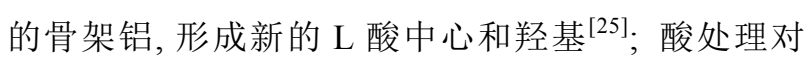
分子篮的骨架铝和非骨架铝脱除非常缓和. 同时, 酸处理及水蒸气处理还可以不同程度地调整分子篮 的孔性质. 对于具有 EUO 拓扑结构这种活性位, 即 铝原子分布特殊的 EU-1 分子篮而言, 脱铝方式对 其物化性质和催化裂解性能的研究至今未见详细报
道. 因此, 本文详细考察了水热处理 $/ \mathrm{HCl}$ 溶液处理 后 EU-1 分子篎的物化性能, 并研究了脱铝方式对 EU-1 分子笁上正己烷裂解反应性能的影响.

\section{1 实验部分}

\section{1 催化剂的制备}

采用本课题组开发的晶种法 ${ }^{[26]}$ 合成 EU-1 分子 篮 $\left(n_{\mathrm{Si}}: n_{\mathrm{Al}}=25\right)$, 所得 $\mathrm{EU}-1$ 原粉经过 $550{ }^{\circ} \mathrm{C}$ 焙烧 6 $\mathrm{h}$ 后, 用 $1.0 \mathrm{~mol} / \mathrm{L}$ 的 $\mathrm{NH}_{4} \mathrm{Cl}$ 溶液按分子篮与交换液 质量比为 $1: 15$ 的比例在 $95^{\circ} \mathrm{C}$ 水浴中交换 $2 \mathrm{~h}$, 然 后过滤, 洗涤, 重复上述过程三次, 得到 $\mathrm{NH}_{4}$-EU-1. 再在 $120^{\circ} \mathrm{C}$ 干燥 $12 \mathrm{~h}, 550{ }^{\circ} \mathrm{C}$ 焙烧得到 $\mathrm{HEU}-1$ 分 子篮.

按分子篮与酸溶液质量比为 $1: 15$ 用 $0.5 \mathrm{~mol} / \mathrm{L}$ 的 $\mathrm{HCl}$ 溶液在 $60^{\circ} \mathrm{C}$ 处理 $\mathrm{HEU}-1$ 分子篮 $2 \mathrm{~h}$, 然后 过滤, 洗涤, 于 $120^{\circ} \mathrm{C}$ 干燥 $12 \mathrm{~h}$ 后, 得到的样品记 为 $\mathrm{HEU}-1(\mathrm{HCl})$.

将 15.0 g HEU-1 分子篎装入反应管 $(\varphi=15$ $\mathrm{mm})$ 中, 在 $\mathrm{N}_{2}$ 气氛下以 $5{ }^{\circ} \mathrm{C} / \mathrm{min}$ 的速度升至 600 ${ }^{\circ} \mathrm{C}$, 在常压, 质量空速为 $1.0 \mathrm{~h}^{-1}$ 的条件下, 通入水蒸 气, 冷却后取出样品. 水热处理时间分别为 2,4 和 $10 \mathrm{~h}$ 的分子篮分别记为 HEU-1(HT2), HEU-1(HT4) 和 HEU-1(HT10)

取 $2.0 \mathrm{~g} \mathrm{HEU}-1$ (HT4) 样品加入到 $0.5 \mathrm{~mol} / \mathrm{L}$ 的 $\mathrm{HCl}$ 溶液中于 $60^{\circ} \mathrm{C}$ 处理 $2 \mathrm{~h}$, 过滤、洗涤后于 120 ${ }^{\circ} \mathrm{C}$ 干燥 $12 \mathrm{~h}$, 所得样品记为 HEU-1(HT4-HCl).

\section{2 催化剂的表征}

采用德国 Bruker D8 X 射线粉末衍射 (XRD) 仪 测定样品的物相结构, $\mathrm{Cu} K_{\alpha}$ 射线, 管压 $40 \mathrm{kV}$, 管流 $30 \mathrm{~mA}$, 扫描范围 $2 \theta=5^{\circ} \sim 50^{\circ}$, 扫描速度 $4.0^{\circ} / \mathrm{min}$. 测样品的结晶度时, 扫描范围 $2 \theta=18^{\circ} \sim 28^{\circ}$, 扫描速 度 $1.0 \%$ min. 按照 Ray 等 ${ }^{[27]}$ 的方法计算 HEU-1 分 子篮的相对结晶度 $\alpha=S_{\mathrm{p}} /\left(S_{\mathrm{p}}+S_{\mathrm{b}}\right)$, 其中 $S_{\mathrm{p}}$ 为 
$18^{\circ} \sim 28^{\circ}$ 之间衍射峰的绝对面积之和, $S_{\mathrm{b}}$ 为 $18^{\circ} \sim 28^{\circ}$ 之间衍射峰背景的面积之和.

采用日本 Rigaku ZSX-100e 型 X 射线荧光仪 (XRF) 分析产品体相硅铝比 $\left(n_{\mathrm{Si}}: n_{\mathrm{Al}}\right)$ 等. 采用美国 麦克公司 ASAP 2020 型自动物理吸附仪测定样品 的比表面积和孔结构. 样品在 $1.3 \mathrm{~Pa}, 350{ }^{\circ} \mathrm{C}$ 净化 处理 $4 \mathrm{~h}$, 并在 $-196{ }^{\circ} \mathrm{C}$ 静态吸附 $\mathrm{N}_{2}$. 比表面积采用 BET 法计算, 利用静态容量法测定样品的孔体积, 采用 $t$-plot 方法计算微孔面积和微孔体积.

样品的 $\mathrm{Al}$ 配位环境信息在 Bruker Advance 400 型固体核磁共振波谱 (NMR) 仪上进行分析. ${ }^{27} \mathrm{Al}$ 的共振频率为 $130.34 \mathrm{MHz}$, 转动频率 $8 \mathrm{kHz}$, 循 环延迟时间 $0.5 \mu \mathrm{s}$. 以 $\mathrm{Al}_{2}\left(\mathrm{SO}_{4}\right)_{3} \cdot 12 \mathrm{H}_{2} \mathrm{O}$ 为标准物.

样品的酸强度分布采用氨气程序升温脱附法 $\left(\mathrm{NH}_{3}-\mathrm{TPD}\right)$ 进行测定, 仪器为自制的程序升温脱附 装置. 首先将 $200 \mathrm{mg}$ 样品在 $600{ }^{\circ} \mathrm{C}, \mathrm{N}_{2}(40 \mathrm{ml} / \mathrm{min})$ 的氛围下净化 $1 \mathrm{~h}$, 然后冷却到 $100{ }^{\circ} \mathrm{C}$, 吸附 $\mathrm{NH}_{3}$ (30 ml/min) $30 \mathrm{~min}$, 并在 $100{ }^{\circ} \mathrm{C}, \mathrm{N}_{2}$ 条件下脱除样 品上物理吸附的 $\mathrm{NH}_{3}$, 之后以 $10^{\circ} \mathrm{C} / \mathrm{min}$ 升温至 600 ${ }^{\circ} \mathrm{C}$, 用北京分析仪器厂 SP-3420 型气相色谱仪记录 $\mathrm{NH}_{3}$-TPD 谱图 (TCD 检测器).

采用吡啶吸附红外光谱 (FT-IR) 法测定样品的 酸量及酸类型。实验在美国 Nicolet 公司 MAGNA-IR 560 型红外光谱仪上进行. 仪器的分辨 率为 $4 \mathrm{~cm}^{-1}$, 精度为 $0.01 \mathrm{~cm}^{-1}$, 扫描次数为 32 次. 压制的自撑片状样品 $(12 \mathrm{mg})$, 先在真空下于 $350^{\circ} \mathrm{C}$ 脱附 $4 \mathrm{~h}$, 然后降至室温吸附吡啶, 最后升温到 200 ${ }^{\circ} \mathrm{C}$ 脱附, $30 \mathrm{~min}$ 后记录谱图.

\section{3 催化剂的评价}

通过正己烷 $(98 \%, \mathrm{AR})$ 催化裂解反应来考察脱 铝 HEU-1 分子篮的催化性能. 采用小型固定床反 应器, 反应管为 $10 \mathrm{~mm} \times 430 \mathrm{~mm}$ 的石英管, 催化剂
装填量为 $1.0 \mathrm{~g}$, 反应条件为 $625^{\circ} \mathrm{C}$, 常压, 正己烷质 量空速 $2.0 \mathrm{~h}^{-1}$. 采用 SP-3420 型气相色谱在线分析, HP-Plot-Q 毛细管柱, FID 检测器.

\section{2 结果与讨论}

\subsection{HCl 溶液处理和水热处理 HEU-1 分子篮的织} 构性质

图 1 是脱铝 HEU-1 分子篮的 XRD 谱. 由图可 见, 所有样品均具有 EU-1 分子篮的特征峰, 说明经 不同方式处理后样品仍然保持 EU-1 分子篮的骨架 结构。经 计算, HEU-1(HCl), HEU-1(HT2), HEU-1 (HT4), HEU-1(HT10) 和 HEU-1(HT4-HCl) 样品的相 对结晶度分别为 $99 \%, 95 \%, 90 \%, 87 \%$ 和 $92 \%$. 由 此可见, 经 $0.5 \mathrm{~mol} / \mathrm{L} \mathrm{HCl}$ 溶液于 $60{ }^{\circ} \mathrm{C}$ 处理 $2 \mathrm{~h}$ 后, $\mathrm{HEU}-1$ 分子篮的结晶度没有明显变化; 而经 $600{ }^{\circ} \mathrm{C}$ 水热处理后, 样品相对结晶度随着水热处理程度的 增加而降低, 与水热处理 $\mathrm{Y}$ 和 ZSM-5 等分子篮的结 果相似 ${ }^{[18,28]}$.

脱铝 HEU-1 分子篎样品的硅铝比列于表 1 . 可

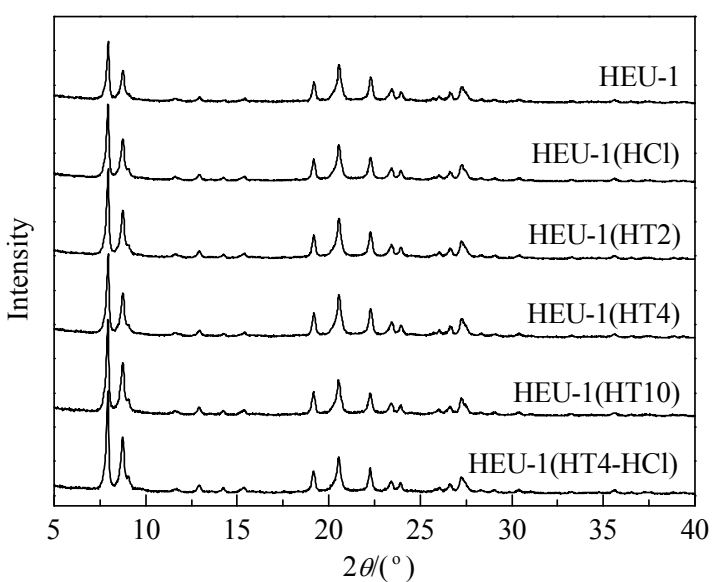

图 $1 \mathrm{HCl}$ 溶液处理和水热处理 HEU-1 分子篮的 XRD 谱

Fig. 1. XRD patterns of HEU-1 zeolites after $\mathrm{HCl}$ and hydrothermal treatments.

表 1 HCI 溶液处理和水热处理 HEU-1 分子篮的织构性质

Table 1 Textural properties of HEU-1 zeolites after $\mathrm{HCl}$ and hydrothermal treatments

\begin{tabular}{|c|c|c|c|c|c|c|c|c|c|c|}
\hline \multirow{2}{*}{ Sample } & \multirow{2}{*}{$n_{\mathrm{Si}} / n_{\mathrm{Al}}{ }^{\mathrm{a}}$} & \multirow{2}{*}{$\alpha^{\mathrm{b} / \%}$} & \multicolumn{3}{|c|}{ Volume $\left(\mathrm{cm}^{3} / \mathrm{g}\right)$} & \multicolumn{3}{|c|}{ Surface area $\left(\mathrm{m}^{2} / \mathrm{g}\right)$} & \multicolumn{2}{|c|}{ Acid amount ${ }^{c}(\mu \mathrm{mol} / \mathrm{g})$} \\
\hline & & & Total & Micropore & Mesopore & BET & Micropore & External & Brönsted & Lewis \\
\hline HEU-1 & 23.1 & 98 & 0.21 & 0.16 & 0.05 & 367 & 310 & 57 & 224 & 187 \\
\hline HEU-1(HCl) & 25.6 & 99 & 0.21 & 0.16 & 0.05 & 361 & 308 & 53 & 219 & 156 \\
\hline HEU-1(HT2) & 24.4 & 95 & 0.22 & 0.15 & 0.07 & 382 & 301 & 81 & 143 & 112 \\
\hline HEU-1(HT4) & 23.7 & 90 & 0.22 & 0.14 & 0.08 & 379 & 289 & 90 & 125 & 119 \\
\hline HEU-1(HT10) & 24.0 & 87 & 0.23 & 0.14 & 0.09 & 383 & 285 & 98 & 108 & 128 \\
\hline HEU-1(HT4-HCl) & 59.3 & 92 & 0.22 & 0.15 & 0.07 & 385 & 290 & 95 & 123 & 67 \\
\hline
\end{tabular}

${ }^{a}$ Determined by XRF. ${ }^{b} \alpha$ is relative crystallinity. ${ }^{c}$ Determined by Py-FTIR at $200{ }^{\circ} \mathrm{C}$. 
以看出, 经 $\mathrm{HCl}$ 溶液处理后, $\mathrm{HEU}-1$ 分子篮的硅铝 比略有增加. 不同于 ZSM-5, MOR 或 $\beta$ 等分子笁, 经 $\mathrm{HCl}$ 溶液处理均能明显改变体相硅铝比的结 果 ${ }^{[19,29]}$. 这可能与 EUO 型分子篎独特的铝原子分 布有关. Peral 等 ${ }^{[3]}$ 采用中子衍射研究证明, 以六甲 双铵为模板剂合成 EU-1 分子篮时, 其 Al 原子定向 分布于 EUO 分子篮十二元环的孔口和底部, 相比于 ZSM-5 和 MOR 等分子篮中随机分布的 $\mathrm{Al}$ 原子, 这 些定向分布的 $\mathrm{Al}$ 原子可能更不易于被酸洗而脱除. 水热处理后 HEU-1 分子篎的硅铝比基本没有变化, 即不改变 HEU-1 分子篮的体相硅铝比, 与 $\mathrm{Y}$, ZSM-5 和 MOR 等分子篮结果相似. 但是, 先水热 处理再 $\mathrm{HCl}$ 溶液处理的 HEU-1 分子篮的硅铝比可 大幅增加到 59.3.

图 2 是各 HEU-1 分子篮样品的 $\mathrm{N}_{2}$ 吸附-脱附 等温线. 可以看出, 与 $\mathrm{HEU}-1$ 分子篎相比, 经 $\mathrm{HCl}$ 溶液直接处理后, HEU-1 分子篮的吸附-脱附等温线 未发生明显变化; 而经水热处理的样品在 $p / p_{0}=$ 0.4 0.9 范围内出现滞后环, 说明 HEU-1 分子篮出

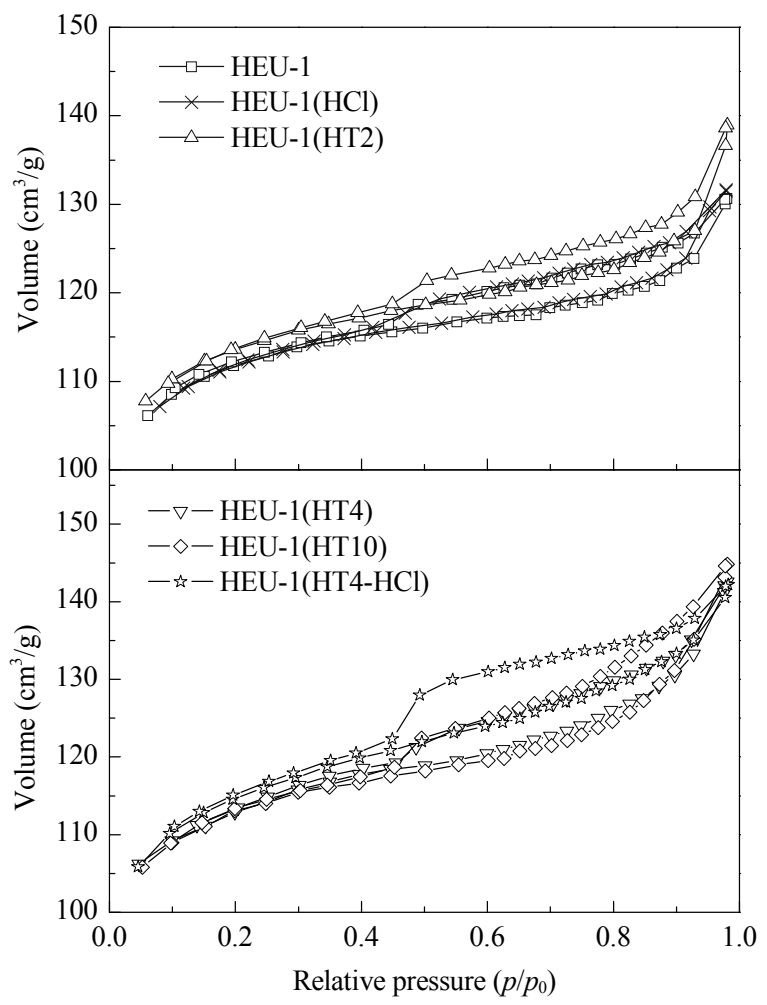

图 $2 \mathrm{HCl}$ 溶液处理和水热处理 HEU-1 分子篮的 $\mathrm{N}_{2}$ 吸附脱附等温线

Fig. 2. $\mathrm{N}_{2}$ adsorption-desorption isotherms of HEU-1 zeolites after $\mathrm{HCl}$ and hydrothermal treatments.
现介孔; 先经水热处理再用 $\mathrm{HCl}$ 溶液处理的 $\mathrm{HEU}-1$ 分子篮也出现滞后环, 且比单一水热处理的样品更 加明显, 表明该样品具有更多的介孔.

各 HEU-1 分子篮样品的织构性质见表 1 . 可以 看出, 经 $\mathrm{HCl}$ 溶液脱铝后, HEU-1 分子篮的孔体积 和比表面积改变不大. 而经水热处理后样品的总孔 体积和介孔体积略有增加, 总比表面积和外比表面 积明显增加, 而微孔体积和微孔面积明显降低, 这是 由于分子篮脱铝形成介孔所致 ${ }^{[19,21,29]}$. 水热处理后 再用 $\mathrm{HCl}$ 溶液处理的 HEU-1 分子篮的孔体积和比 表面积与单一水热处理的基本相同.

图 3 是脱铝 HEU-1 分子篮的 ${ }^{27} \mathrm{Al}$ MAS NMR 谱. 由图可见, 未处理的 HEU-1 分子篮在 $\delta=54$ 和 0 附近分别出现一个强的尖峰和弱的宽峰, 分别对 应于四配位的骨架铝和六配位的非骨架 铝 ${ }^{[22]}$; HEU-1 $(\mathrm{HCl})$ 分子篮在 $\delta=0$ 附近弱的宽峰基本消失, 说明经 $0.5 \mathrm{~mol} / \mathrm{L}$ 的 $\mathrm{HCl}$ 溶液处理后, 可以完全脱 除分子篮的非骨架铝, 且对骨架铝基本没有影响, XRF 法测得 HEU-1 $(\mathrm{HCl})$ 的硅铝比略有增加. 随着 水热处理程度的增加, HEU-1 分子篮的六配位非骨 架铝信号 $(\delta=0)$ 逐渐增强, 而四配位骨架铝的信号 $(\delta=54)$ 逐渐降低, 说明水热处理可以将 HEU-1 分 子笁的骨架铝从骨架脱除而变成非骨架铝, 但分子 篮体相的硅铝比没有明显变化. 可见, 脱除下来的 铝仍然留在分子篮的体相中. 还可以看出, 先水热 处理 $4 \mathrm{~h}$ 再经过 $0.5 \mathrm{~mol} / \mathrm{L} \mathrm{HCl}$ 处理后, $\mathrm{HEU}-1$ 分子 篮的非骨架铝信号几乎全部消失, 说明样品的非骨

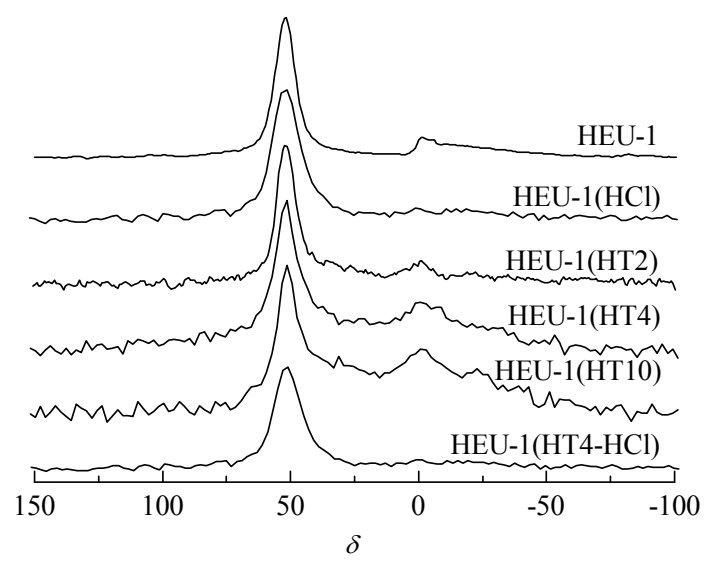

图 $3 \mathrm{HCl}$ 溶液处理和水热处理 $\mathrm{HEU}-1$ 分子篮的 ${ }^{27} \mathrm{Al}$ MAS NMR 谱

Fig. 3. ${ }^{27} \mathrm{Al}$ MAS NMR spectra of dealuminated HEU-1 zeolites after $\mathrm{HCl}$ and hydrothermal treatments. 
架铝物种被脱除, 其硅铝比由 23.1 增加到 59.3. 相 比于骨架铝物种, 非骨架铝物种更容易通过酸洗而 脱除 ${ }^{[30]}$. 因此, 先水热处理后再用 $\mathrm{HCl}$ 溶液处理可 以明显提高 HEU-1 分子篮的骨架硅铝比.

综上可知, $\mathrm{HCl}$ 溶液单独处理只能脱除 HEU-1 分子篮上的非骨架铝, 但不能有效脱除骨架铝, 因而 样品的硅铝比增加不大, 可见 EU-1 分子篮对酸体 系具有很强的稳定性; $600{ }^{\circ} \mathrm{C}$ 的高温水热处理可以 将 HEU-1 分子篮上的骨架铝脱除而变成非骨架铝, 再经过 $\mathrm{HCl}$ 溶液处理可以脱除非骨架铝, 从而提高 HEU-1 分子篮骨架硅铝比, 达到优化 HEU-1 分子篮 酸分布的目的.

\section{$2.2 \mathrm{HCl}$ 溶液处理和水热处理 HEU-1 分子篎的酸 性质}

图 4 是各脱铝 HEU-1 分子篮的 $\mathrm{NH}_{3}$-TPD 谱. 图中 $200{ }^{\circ} \mathrm{C}$ 左右的 $\mathrm{NH}_{3}$ 的脱附峰对应了 $\mathrm{HEU}-1$ 分 子篮表面的弱酸位; $350{ }^{\circ} \mathrm{C}$ 左右的 $\mathrm{NH}_{3}$ 脱附峰对应 于强酸位 ${ }^{[31]}$. 由图可见, $\mathrm{HEU}-1$ 分子篮经 $\mathrm{HCl}$ 溶液 处理后, 其强酸中心的强度和数量几乎不变, 但弱酸 中心数量减少. 这是由于 $\mathrm{HCl}$ 溶液的处理只能脱除 分子篮非骨架铝造成的, 与 ${ }^{27} \mathrm{Al}$ MAS NMR 结果一 致. 经水热处理后, HEU-1 样品的强酸中心和弱酸 中心数量均明显降低, 且随着水热处理程度的增加, 强酸中心数量减少更加明显。这与 ZSM-5 水热脱 铝有着相似的规律 ${ }^{[16,32]}$. 先经水热处理再经 $\mathrm{HCl}$ 溶 液处理, HEU-1 分子笁的强酸中心和弱酸中心数量

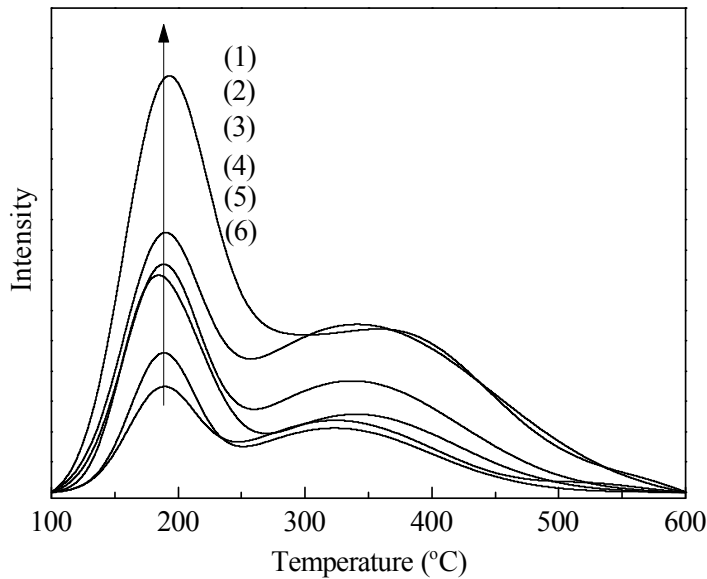

图 $4 \mathrm{HCl}$ 处理和水热处理 $\mathrm{HEU}-1$ 分子篎的 $\mathrm{NH}_{3}$-TPD 谱

Fig. 4. $\mathrm{NH}_{3}$-TPD profiles of dealuminated HEU-1 zeolites after $\mathrm{HCl}$ and hydrothermal treatments. (1) HEU-1; (2) HEU-1(HCl); (3) HEU-1(HT2); (4) HEU-1(HT4); (5) HEU-1(HT10); (6) HEU-1(HT-HCl).
也均明显降低, 主要是因为水热处理后, HEU-1 分 子篮的部分 $\mathrm{Al}$ 原子从骨架中脱除变为非骨架 $\mathrm{Al}$, 且后者易被 $\mathrm{HCl}$ 溶液洗涤而脱除. 由此可见, 相比 于单一处理方式, 水热处理后再用 $\mathrm{HCl}$ 溶液处理的 方法既可以对 HEU-1 分子篮的弱酸中心进行修饰, 也可以对强酸中心进行修饰, 从而达到同时调变 HEU-1 分子笁酸强度和酸密度的目的.

图 5 是 $\mathrm{HCl}$ 溶液处理和水热处理 $\mathrm{HEU}-1$ 分子 篎的吡啶吸附 FT-IR 谱. 1545 和 $1450 \mathrm{~cm}^{-1}$ 处的吸 收峰分别归属于分子笁上 Brönsted 酸中心和 Lewis 酸中心; 而 $1490 \mathrm{~cm}^{-1}$ 处吸收峰则是 Brönsted 酸中 心和 Lewis 酸中心共同作用的结果 ${ }^{[33] ;} 1545$ 和 $1450 \mathrm{~cm}^{-1}$ 处吸收峰的强度分别反映了分子篮的 Brönsted 酸量和 Lewis 酸量. 由图可见, 经各方式处 理后 HEU-1 分子篎的酸量明显降低 (1545 和 1450 $\left.\mathrm{cm}^{-1}\right)$, 与 $\mathrm{NH}_{3}-\mathrm{TPD}$ 结果一致. 根据 Emeis 等 ${ }^{[34]}$ 研究 的吸光系数计算得各样品的 Brönsted 和 Lewis 酸量 列于表 1. 可以看出, 与 HEU-1 分子篮相比, HEU-1 $(\mathrm{HCl})$ 样品的 Brönsted 酸量基本保持不变, 而 Lewis 酸量则由 $187 \mu \mathrm{mol} / \mathrm{g}$ 降至 $156 \mu \mathrm{mol} / \mathrm{g}$. 随着 水热处理程度的增加, HEU-1 分子篮的 Brönsted 酸 量逐渐降低, Lewis 酸量逐渐增加. 与 HEU-1(HT-4) 相比, HEU(HT4-HCl) 的 Brönsted 酸量基本保持不 变, 而 Lewis 酸量由 $119 \mu \mathrm{mol} / \mathrm{g}$ 显著降到 $67 \mu \mathrm{mol} / \mathrm{g}$, 与 ${ }^{27} \mathrm{Al} \mathrm{NMR}$ 结果一致. 由此可见, $\mathrm{HCl}$ 溶液处理对

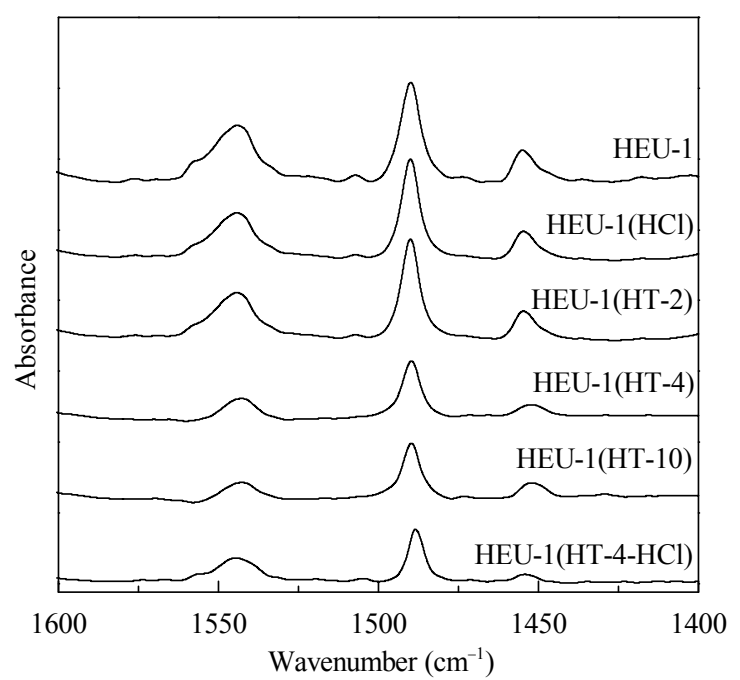

图 $5 \mathrm{HCl}$ 处理和水热处理 HEU-1 分子篮吸附吡啶的 FT-IR 谱

Fig. 5. Pyridine adsorbed FT-IR spectra of HEU-1 zeolites after $\mathrm{HCl}$ and hydrothermal treatments at $200{ }^{\circ} \mathrm{C}$. 
HEU-1 分子篎的 Brönsted 酸量影响不大, 但可以明 显调变 HEU-1 分子篮的 Lewis 酸量; 而水热处理 可以明显调节 HEU-1 分子篮的 Brönsted 酸量; 水 热与 $\mathrm{HCl}$ 溶液处理结合可以达到明显调变 HEU-1 分子篮 Brönsted 酸和 Lewis 酸量的目的, 不同于 $\beta$ 分子篮单独使用酸洗涤即可调节酸性的结果 ${ }^{[30]}$.

\section{$2.3 \mathrm{HCl}$ 溶液处理和水热处理 HEU-1 分子篮的催 化裂解性能}

在 $625^{\circ} \mathrm{C}$, 正己烷质量空速为 $2.0 \mathrm{~h}^{-1}$ 的反应条 件下, 各 HEU-1 分子篮样品上正己烷裂解反应结果 见表 2. 可以看出, 与 HEU-1 分子篮相比, HEU- $1(\mathrm{HCl})$ 上正己烷转化率略有降低, 而水热处理 的 HEU-1(HT2), HEU-1(HT4) 和 HEU-1(HT10) 上 正己烷转化率明显降低, 且随着水热处理程度的增 加, 样品活性逐渐下降. 另外, HEU-1(HT4) 和 HEU-1(HT4-HCl) 样品活性接近, 结合酸性表征结 果可认为, 分子篎活性与其 Brönsted 酸量成正比.

对于低碳烯烃选择性, HEU-1 分子篮经 $\mathrm{HCl}$ 溶 液处理后, 丙烯的选择性从 $21.9 \%$ 略增至 23.7\%; 而经水热处理后, 样品上丙烯选择性则显著增至 $31.4 \%$. 这主要是由于经水热处理后, HEU-1 分子篮 的酸密度降低, 从而抑制了催化裂解反应中二次反 应, 尤其是氢转移反应的发生. 另外, 水热处理后再 经 $\mathrm{HCl}$ 溶液处理的 HEU-1 分子篮, 表现出最高的丙 烯选择性, 达 $35.2 \%$. 这是因为水热处理脱除了 HEU-1 分子篎的骨架铝物种, 明显降低了分子篮的 酸密度和酸强度, 再经 $\mathrm{HCl}$ 溶液处理脱除了非骨架 铝物种, 从而降低了 Lewis 酸量, 疏通了分子篮的孔 道, 使得分子篮的扩散性能提高. Inagaki 等 ${ }^{[35]}$ 同样 发现, 经过水热处理的 MCM-68 分子篮上正已烷裂 化反应的丙烯选择性大幅度提高, 这与 MCM-68 分 子篎酸密度和酸强度的减少有密切的关系.
正己烷催化裂解是典型的平行反应, 丙烯是中

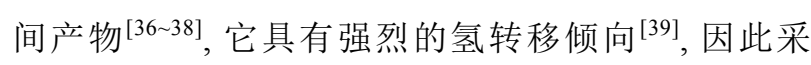
用丙烷/丙烯来表征反应氢转移反应的程度, 结果如 图 6 所示. 可以看出, HEU-1 分子篮经 $\mathrm{HCl}$ 溶液处 理或水热处理 $2 \mathrm{~h}$ 后, 丙烷/丙烯比略有降低, 维持在 0.75 0.80 之间; 经水热处理 $4 \mathrm{~h}$ 后, HEU-1 分子篮 上的丙烷/丙烯比降到 0.49 , 且随着水热处理程度的 进一步增加而降至 0.32. HEU-1(HT4-HCl) 分子篮 上丙烷/丙烯比可达 0.27. 丙烷/丙烯比值的降低说 明该分子篮的氢转移能力大幅降低, 从而使得丙烯 选择性大幅提高. 分子篮的酸密度大有利于氢转移 反应的发生, 而通过水热和 $\mathrm{HCl}$ 溶液处理降低了 HEU-1 分子篮的酸密度, 抑制了氢转移反应的发生, 从而提高了丙烯选择性.

各 HEU-1 分子篮上正己烷裂解反应中正己烷 转化率随反应时间的变化如图 7 所示. 可以看出,

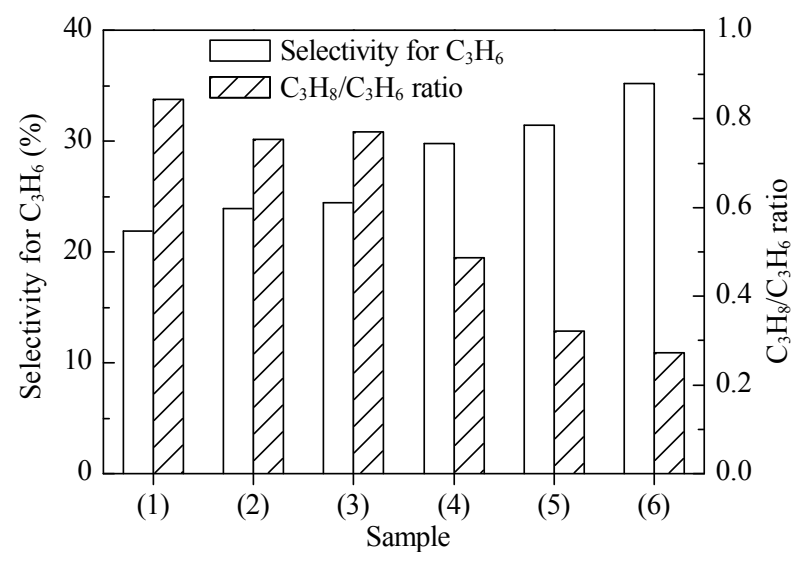

图 $6 \mathrm{HCI}$ 溶液处理和水热处理 HEU-1 分子篮的丙烯选择 性和氢转移性能

Fig. 6. Propylene selectivity and hydrogen transfer properties on HEU-1 zeolites after $\mathrm{HCl}$ and hydrothermal treatments (1) HEU-1; (2) HEU-1(HCl); (3) HEU-1(HT2); (4) HEU-1(HT4); (5) HEU-1(HT10); (6) HEU-1(HT4-HCl). Reaction conditions: catalyst $1.0 \mathrm{~g}, 625^{\circ} \mathrm{C}$, $n\left(\mathrm{~N}_{2}\right): n(n$-hexane $)=5: 1, \mathrm{WHSV}=2.0 \mathrm{~h}^{-1}, \mathrm{TOS}=15 \mathrm{~min}$.

表 $2 \mathrm{HCl}$ 处理和水热处理 HEU-1 分子篮的正己烷催化裂解性能

Table 2 Catalytic cracking performance of $n$-hexane of HEU-1 zeolites by $\mathrm{HCl}$ and hydrothermal treatments

\begin{tabular}{lcrrrrrrrr}
\hline \multirow{2}{*}{ Sample } & Conversion & \multicolumn{9}{c}{ Selectivity (\%) } \\
\cline { 2 - 9 } & $(\%)$ & $\mathrm{CH}_{4}$ & $\mathrm{C}_{2} \mathrm{H}_{6}$ & $\mathrm{C}_{2} \mathrm{H}_{4}$ & $\mathrm{C}_{3} \mathrm{H}_{8}$ & $\mathrm{C}_{3} \mathrm{H}_{6}$ & $\mathrm{C}_{4} \mathrm{H}_{10}$ & $\mathrm{C}_{4} \mathrm{H}_{8}$ & $\mathrm{C}_{5+}$ \\
\hline HEU-1 & 99.4 & 10.5 & 12.0 & 24.6 & 18.5 & 21.9 & 3.5 & 4.1 & 4.9 \\
HEU-1(HCl) & 97.8 & 8.3 & 11.2 & 23.3 & 18.0 & 23.7 & 4.8 & 6.3 & 4.4 \\
HEU-1(HT2) & 89.6 & 6.8 & 9.3 & 23.2 & 18.8 & 24.4 & 6.1 & 6.7 & 4.7 \\
HEU-1(HT4) & 85.7 & 5.6 & 7.8 & 22.4 & 14.5 & 29.8 & 7.5 & 8.6 & 3.8 \\
HEU-1(HT10) & 80.2 & 4.5 & 6.9 & 19.3 & 10.1 & 31.4 & 10.0 & 14.6 & 3.2 \\
HEU-1(HT4-HCl) & 84.1 & 3.4 & 5.9 & 16.3 & 9.6 & 35.2 & 10.5 & 16.1 & 3.0 \\
\hline
\end{tabular}

Reaction conditions: $625^{\circ} \mathrm{C}, n\left(\mathrm{~N}_{2}\right): n(n$-hexane $)=5: 1, \mathrm{WHSV}=2.0 \mathrm{~h}^{-1}, \mathrm{TOS}=15 \mathrm{~min}$. 


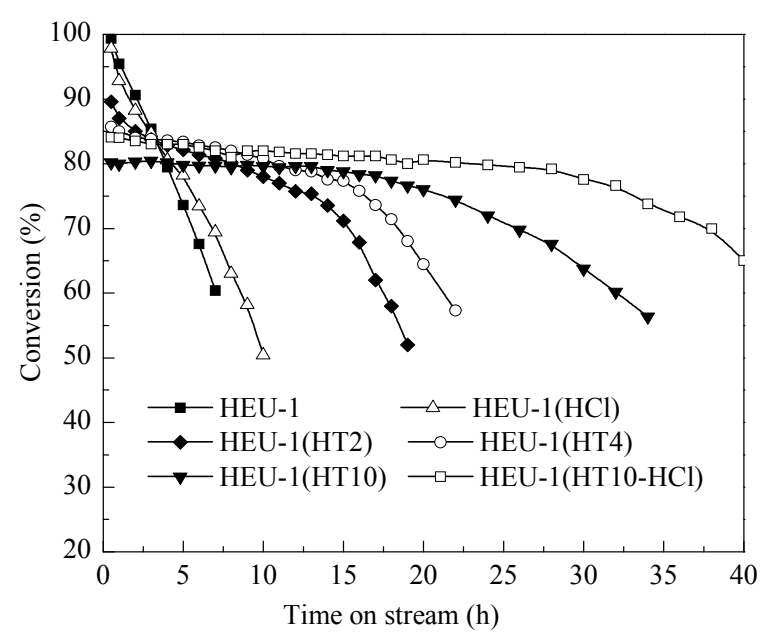

图 $7 \mathrm{HCl}$ 处理和水热处理 HEU-1 分子篮的正己烷转化率 随反应时间的变化

Fig. 7. Relationship between $n$-hexane and time for HEU-1 zeolites after hydrochloric acid and hydrothermal treatments. Reaction conditions: catalyst $1.0 \mathrm{~g}, 625^{\circ} \mathrm{C}, n\left(\mathrm{~N}_{2}\right): n(n$-hexane $)=5: 1$, WHSV $=2.0$ $\mathrm{h}^{-1}$.

随着 HEU-1 分子篮强酸中心减少, 初始活性明显降 低. 在烷烃催化裂解反应中催化剂的失活主要是由

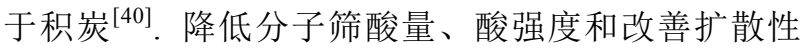
能均可以延长分子篮的寿命. 强酸量越少, HEU-1 分子篎的活性和稳定性越高. 经 $\mathrm{HCl}$ 溶液处理后, HEU-1 分子篮稳定性略有提高; 而经水热处理后, HEU-1 分子篮的稳定性明显改善, HEU-1(HT2), HEU-1(HT4) 和 HEU-1(HT10) 分子篮上正己烷转化 率达到 $60 \%$ 经历的时间由 $8 \mathrm{~h}$ 分别增至 18,20 和 $30 \mathrm{~h}$. 另外, HEU-1 分子篮先经水热处理再经 $\mathrm{HCl}$ 溶液处理后, 反应 $30 \mathrm{~h}$ 内正已烷转化率保持在 $80 \%$ 以上, $40 \mathrm{~h}$ 时仍高于 $60 \%$. 对比 HEU-1(HT4) 和 HEU-1(HT4-HCl) 可以发现, 分子耖上的 Lewis 酸中 心对稳定性也有影响, 尤其是强 Lewis 酸中心. 烷 烃吸附在 Lewis 酸中心上很难脱离, 最终形成焦炭 而使催化剂结焦失活 ${ }^{[41]}$. 另外, 脱铝 HEU-1 分子篮 上产生了介孔, 有利于产物和积炭前驱物的扩散, 进 一步提高了 HEU-1 分子篮的稳定性.

各 HEU-1 分子篮上正己烷裂解反应的乙烯和 丙烯选择性随正己烷转化率的变化如图 8 所示. 可 以看出, 正己烷转化率相同时, $\mathrm{HEU}-1$ 分子篮经 $\mathrm{HCl}$ 处理后, 相应地乙烯的择性性略有下降, 丙烯选择性 略有提高; 经水热处理的 HEU-1(HT-2) 和 HEU-1(HT-4) 样品上, 乙烯和丙烯选择性变化不大,

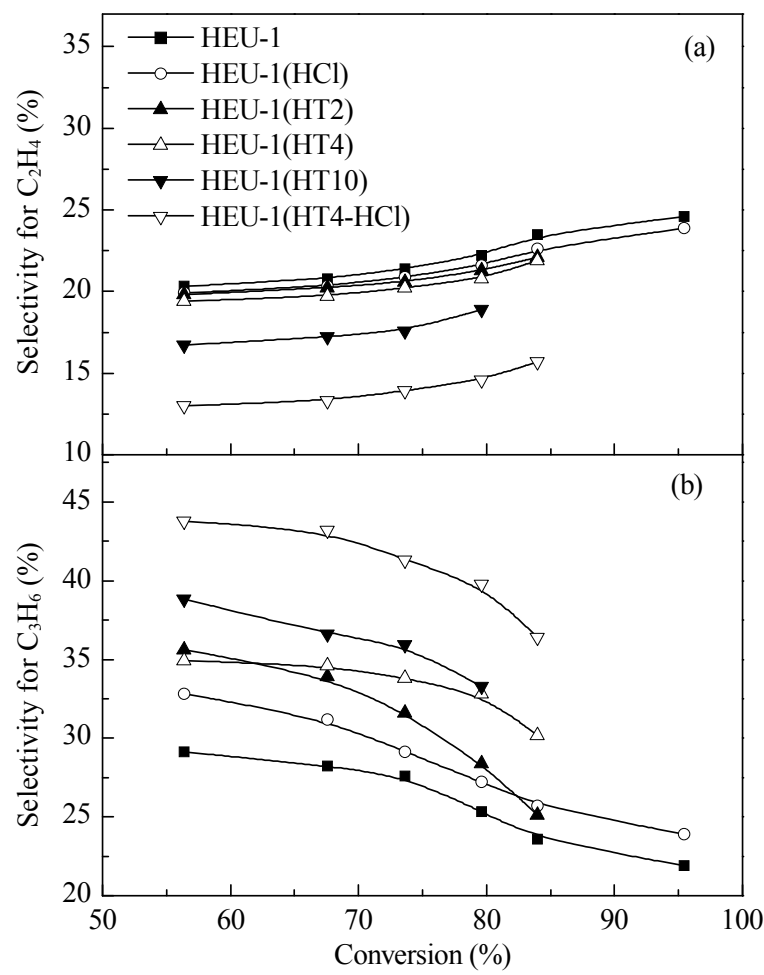

图 $8 \mathrm{HCl}$ 溶液处理和水热处理 HEU-1 分子篮上乙烯和丙 烯选择性随转化率的变化

Fig. 8. Relationship between selectivities for ethylene (a) and propylene (b) and $n$-hexane conversion. Reaction conditions: catalyst 1.0 $\mathrm{g}, 625^{\circ} \mathrm{C}, n\left(\mathrm{~N}_{2}\right): n(n$-hexane $)=5: 1, \mathrm{WHSV}=2.0 \mathrm{~h}^{-1}$.

而经水热处理 $10 \mathrm{~h}$ 的 HEU-1(HT-10) 样品上乙烯的 选择性明显下降，丙烯的选择明显提高; HEU-1(HT-4-HCl) 样品具有更低的乙烯选择性和更 高的丙烯选择性.

综上所述, 随着 HEU-1 分子篮酸密度减少, 低 碳烯烃的初始选择性和稳定性增加, 但是分子笚的 活性降低. 因此,一般在 $600{ }^{\circ} \mathrm{C}$ 水热处理 $4 \mathrm{~h}$ 为宜, 以控制脱铝程度. 水热处理与 $\mathrm{HCl}$ 溶液处理两种脱 铝方式相结合可以有效调节 HEU-1 分子篮的酸性, 从而使其在正己烷催化裂解反应中表现出较高的丙 烯选择性.

\section{3 结论}

对 HEU-1 分子篮分别进行 $\mathrm{HCl}$ 溶液处理、水热 处理和两种方法结合的脱铝研究. $\mathrm{HCl}$ 溶液处理只 能脱除 HEU-1 分子篮的非骨架铝, 调节 HEU-1 分 子篮的弱酸中心; 水热处理可以使 HEU-1 分子篮 骨架发生脱铝, 主要调节 EU-1 分子篎的强酸中心. 
对 HEU-1 分子篮先进行水热处理, 再用 $\mathrm{HCl}$ 溶液洗 涤, 可以使 HEU-1 分子笁的强酸与弱酸中心均得到 调节, 同时产生部分介孔, 使得 HEU-1 分子篮的酸 性质和扩散性能均得到改善. 在催化裂解正己烷制 丙烯的反应中, 水热处理与 $\mathrm{HCl}$ 溶液处理相结合制 备的脱铝 HEU-1 分子篮上的氢转移反应大幅降低, 因而表现出了较高的丙烯收率和选择性, 分别比未 改性的 HEU-1 分子篮高 7.8\% 和 13.3\%, 其稳定性 也得到了明显提高.

\section{参 考 文 献}

1 Casci J L, Lowe B M, Whittam T V. EP 0042 226. 1981

2 Briscoe N A, Johnson D W, Shannon M D, Kokotailo G T, McCusker L B. Zeolites, 1988, 8: 74

3 Peral I, Jones C Y, Varkey S P, Lobo R F. Microporous Mesoporous Mater, 2004, 71: 125

4 Moreau F, Moreau P, Gnep N S, Magnoux P, Lacombe S, Guisnet M. Microporous Mesoporous Mater, 2006, 90: 327

5 Alario F, Joly J-F, Magne-Drisch J, Merlen E, Benazzi E, Lacombe S. US 6337427B1. 2002

6 Joly J-F, Coupard V, Magne-Drisch J, Alario F, Merlen E, Lacombe S, Benazzi E. US 6147 269. 2000

7 Teketel S, Skistad W, Benard S, Olsbye U, Lillerud K P, Beato P, Svelle S. ACS Catal, 2011, 2: 26

8 Abrevaya H. Stud Surf Sci Catal, 2007, 170: 1244

9 Jolly S, Saussey J, Bettahar M M, Lavalley J C, Benazzi E. Appl Catal A, 1997, 156: 71

10 Corma A, Melo F V, Sauvanaud L, Ortega F. Catal Today, 2005, 107-108: 699

11 Kotrel S, Knözinger H, Gates B C. Microporous Mesoporous Mater, 2000, 35-36: 11

12 Corma A, Mengual J, Miguel P J. Appl Catal A, 2012, 421: 121

13 Teimouri Sendesi S M, Towfighi J, Keyvanloo K. Catal Commum, 2012, 27: 114

14 冯利利, 齐兴义, 李征, 朱岳麟, 李星国. 催化学报 (Feng L L, Qi X Y, Li Zh, Zhu Y L, Li X G. Chin J Catal), 2012, 30: 340

15 Lee K-H, Ha B-H. Microporous Mesoporous Mater, 1998, 23: 211

16 Triantafillidis C S, Vlessidis A G, Nalbandian L, Evmiridis N P. Microporous Mesoporous Mater, 2001, 47: 369

17 Wei R C, Li C Y, Yang C H, Shan H H. J Nat Gas Chem, 2011, 20: 261

18 Jin L J, Hu H Q, Zhu S W, Ma B. Catal Today, 2010, 149: 207

19 李强, 窦涛, 霍全, 徐庆虎, 巩雁军, 潘慧芳. 物理化学 学报 (Li Q, Dou T, Huo Q, Xu Q H, Gong Y J, Pan H F. Acta Phys-Chim Sin), 2008, 24: 1192

20 Müller M, Harvey G, Prins R. Microporous Mesoporous Mater, 2000, 34: 135

21 Marques J P, Gener I, Lopes J M, Ribeiro F R, Guisnet M.
Appl Catal A, 2006, 301: 96

22 Fan Y, Bao X, Lin X, Shi G, Liu H. J Phys Chem B, 2006, 110: 15411

23 龙化云, 王祥生, 孙万付, 刘海鸥, 程晓晶. 石油学报 (石油加工)(Long H Y, Wang X Sh, Sun W F, Liu H O, Cheng X J. Acta Petrol Sin (Petrol Process Sect)), 2009, 25: 332

24 张培青, 王祥生, 郭洪臣, 徐舟波, 赵乐平, 胡永康. 催 化学报 (Zhang P Q, Wang X Sh, Gou H Ch, Xu Zh B, Zhao L P, Hu Y K. Chin J Catal), 2003, 24: 904

25 Zhao X, Guo X, Wang X. Fuel Process Technol, 2007, 88: 237

26 Xu Q H, Gong Y J, Xu W J, Xu J, Deng F, Dou T. J Colloid Interface Sci, 2011, 358: 252

27 Ray P K, Montague P E. J Appl Polym Sci, 1977, 21: 1267

28 Xu B, Sievers C, Hong S B, Prins R, van Bokhoven J A. J Catal, 2006, 244: 163

29 Li X, Prins R, van Bokhoven J A. J Catal, 2009, 262: 257

30 毛东森, 郭强胜, 孟涛, 卢冠忠. 物理化学学报 (Mao D S, Guo Q Sh, Meng T, Lu G Zh. Acta Phys-Chim Sin), 2010, 26: 338

31 Costa C, Dzikh I P, Lopes J M, Lemos F, Ribeiro F R. J Mol Catal A, 2000, 154: 193

32 Sano T, Uno Y, Wang Z B, Ahn C H, Soga K. Microporous Mesoporous Mater, 1999, 31: 89

33 Barzetti T, Selli E, Moscotti D, Forni L. J Chem Soc, Faraday Trans, 1996, 92: 1401

34 Emeis C A. J Catal, 1993, 141: 347

35 Inagaki S, Takechi K, Kubota Y. Chem Commun, 2010, 46: 2662

36 Bortnovsky O, Sazama P, Wichterlova B. Appl Catal A, 2005, 287: 203

37 Lukyanov D B, Shtral V I, Khadzhiev S N. J Catal, 1994, 146: 87

38 Riekerti L, Zhou J Q. J Catal, 1992, 137: 437

39 朱向学, 宋月芹, 李宏冰, 刘盛林, 孙新德, 徐龙伢. 催 化学报 (Zhu X X, Song Y J, Li H B, Liu Sh L, Sun X D, Xu L Y. Chin J Catal), 2005, 26: 111

40 Corma A, González-Alfaro V, Orchillés A V. Appl Catal A, 1999, 187: 245

41 Corma A, Planelles J, Sánchez-Marín J, Tomás F. J Catal, 1985, 93: 30

\section{英 译 文 \\ English Text}

Zeolite EU-1 was first synthesized by Casci et al. in 1981 . It has a one-dimensional channel system with 10 -memberedring $(\mathrm{MR})$ openings running along the direction, connected to side-pockets by 12 MRs in the $(001)$ direction [1,2]. It has been reported that when EU-1 zeolite is prepared from active aluminosilicate gels using hexamethonium (HM) ions as the structure-directing agents, the framework aluminum species are anchored at the bottoms and pore mouths of the 12-MR 
side-pockets $[3,4]$, that is, the active sites of the EU-1 zeolite are located at the bottoms and pore mouths of the 12-MR side-pockets. Because of its peculiar pore architecture and chemical properties, zeolite EU-1 has applications in the isomerization of $\mathrm{C} 8$ aromatics. It has higher activity and selectivity in xylene isomerization reactions than do other zeolites such as ZSM-5, BEA, and mordenite (MOR) zeolites $[5,6]$. Teketel et al. [7] have studied the performance of EU-1 zeolite in methanol to hydrocarbons reactions. It has been found that EU-1 zeolite showed excellent activity, including hydrogen transfer activity, leading to lower olefins and higher aromatics. Abrevaya et al. [8] found that EU-1 zeolite $\left(n_{\mathrm{Si}}: n_{\mathrm{Al}}=25-29\right)$ has good catalytic cracking properties for naphtha. However, a low selectivity for light olefins was found as a result of an excess of acid sites, which caused extensive hydrogen transfer reactions. The selectivity for ethylene plus propylene was only $40 \%$; the selectivities for propane and propylene were almost the same.

The key to increasing the selectivity for light olefins is limiting hydrogen transfer reactions in paraffin cracking [9-14]. Reducing the acid density is an important method of enhancing the selectivity for light olefins [15-17]. The use of acid treatments and hydrothermal treatments as effective methods of reducing the acid densities of zeolites has attracted widespread research interest [15,16,18-24]. For FAU, ZSM-5, BEA, and MOR zeolites, the framework aluminum species can be removed effectively by hydrothermal treatment, and extra Lewis acid sites and hydroxyl groups are formed [25]. Both the framework and extra-framework aluminum species can be removed by acid treatment. The pore properties are also adjusted by acid and hydrothermal treatments. So, in this work, the influence of acid and hydrothermal treatments on the textural properties and cracking performance of HEU-1 zeolite was studied. The effect of hydrothermal treatment on the acidity and structure of HEU-1 zeolite was systematically investigated and the $n$-hexane catalytic cracking performance of dealuminated EU-1 zeolite was also studied.

\section{Experimental}

\subsection{Catalyst synthesis}

EU-1 zeolite was synthesized using the seeding method described in Ref. [26] and was then calcined at $550{ }^{\circ} \mathrm{C}$ for 6 h. The calcined EU-1 zeolite was treated with $1.0 \mathrm{~mol} / \mathrm{L}$ $\mathrm{NH}_{4} \mathrm{Cl}$ solution $(15 \mathrm{ml} / \mathrm{g})$ for $2 \mathrm{~h}$ at $95{ }^{\circ} \mathrm{C}$, washed with distilled water, dried at $120{ }^{\circ} \mathrm{C}$ for $12 \mathrm{~h}$, and then calcined at $550{ }^{\circ} \mathrm{C}$ for $6 \mathrm{~h}$. After repeating the above process three times, HEU-1 zeolite was obtained.

HEU-1 zeolite was heated to $600{ }^{\circ} \mathrm{C}$ in a $\mathrm{N}_{2}$ atmosphere at a heating rate of $5{ }^{\circ} \mathrm{C} / \mathrm{min}$. At this temperature, steam was passed over the sample at a weight hourly space velocity (WHSV) of $1.0 \mathrm{~h}^{-1}$ for 2,4 , and $10 \mathrm{~h}$, and the obtained samples were denoted by HEU-1(HT2), HEU-1(HT4), and HEU-1(HT10), respectively.

The HEU-1 and HEU-1(HT4) zeolites were each treated in a $0.5 \mathrm{~mol} / \mathrm{L} \mathrm{HCl}$ solution $(15 \mathrm{ml} / \mathrm{g})$ for $2 \mathrm{~h}$ at $60{ }^{\circ} \mathrm{C}$, and then filtered, washed with distilled water, and dried at $120^{\circ} \mathrm{C}$ for $12 \mathrm{~h}$. The obtained samples were denoted by HEU-1(HCl) and HEU-1(HT4-HCl).

\subsection{Catalyst characterization}

X-ray diffraction (XRD) patterns were recorded on a Shimadzu XRD-6000 diffractometer using $\mathrm{Cu} K_{\alpha}$ radiation operated at $40 \mathrm{kV}$ and $30 \mathrm{~mA}$ with a $2 \theta$ scanning speed of $4 \% \mathrm{~min}$. The scattering data were collected in a continuous scanning mode from $18^{\circ}$ to $28^{\circ}$ at a scanning rate of $1 \%$ min to determine the crystallinity. As described by Ray et al. [27], the sample crystallinity was evaluated according to the formula: $\alpha=S_{\mathrm{p}} /\left(S_{\mathrm{p}}+S_{\mathrm{b}}\right)$, where $S_{\mathrm{p}}$ is the net area of diffracted peaks between $2 \theta$ values of $18^{\circ}$ and $28^{\circ}$, and $S_{\mathrm{b}}$ is the diffracted peaks background area.

The bulk chemical compositions were measured on a Rigaku ZSX-100e X-ray fluorescence (XRF) analyzer. Nitrogen adsorption-desorption isotherms were measured at -196 ${ }^{\circ} \mathrm{C}$ using an ASAP 2020 instrument. Prior to adsorption measurements, the samples were evacuated at $350{ }^{\circ} \mathrm{C}$ for $4 \mathrm{~h}$. The specific surface area was calculated using the Brunauer-Emmett-Teller (BET) method, the pore volume was calculated using the static capacity method, and the microporous specific surface area and volume were calculated using the $t$-plot method.

${ }^{27} \mathrm{Al}$ magic-angle spinning nuclear magnetic resonance (MAS NMR) experiments were performed on a Bruker Advance 400 spectrometer. The ${ }^{27} \mathrm{Al}$ MAS NMR spectra were recorded at a resonance frequency of $130.34 \mathrm{MHz}$, a spinning rate of $8 \mathrm{kHz}$, and a pulse length of $0.5 \mu \mathrm{s}$. The ${ }^{27} \mathrm{Al}$ MAS NMR chemical shifts were referenced to $\mathrm{Al}_{2}\left(\mathrm{SO}_{4}\right)_{3} \cdot 12 \mathrm{H}_{2} \mathrm{O}$.

The acid strength distribution was investigated using ammonia temperature-programmed desorption $\left(\mathrm{NH}_{3}-\mathrm{TPD}\right)$ equipment. The $\mathrm{NH}_{3}$ concentration was detected using a thermal conductivity detector. The samples were pretreated at $600{ }^{\circ} \mathrm{C}$ for $1 \mathrm{~h}$ in a $\mathrm{N}_{2}$ stream at a flow rate of $40 \mathrm{ml} / \mathrm{min}$, and then cooled to $100{ }^{\circ} \mathrm{C}$. After $\mathrm{NH}_{3}$ adsorption, the samples were evacuated at $100{ }^{\circ} \mathrm{C}$ to remove physically adsorbed $\mathrm{NH}_{3}$, heated to $600{ }^{\circ} \mathrm{C}$ at a rate of $10{ }^{\circ} \mathrm{C} / \mathrm{min}$, and then the spectrogram was recorded.

The Brönsted and Lewis acid sites of the samples were investigated using a Nicolet MAGNA-IR 560 infrared spectrometer (FT-IR) based on pyridine adsorption. The test conditions were $4 \mathrm{~cm}^{-1}$ distinguishability, $0.01 \mathrm{~cm}^{-1}$ accuracy, and 32 scans. Self-supporting wafers of the samples 
were pretreated at $350{ }^{\circ} \mathrm{C}$ for $4 \mathrm{~h}$ under vacuum, and then cooled to room temperature. After pyridine adsorption, heating to $200{ }^{\circ} \mathrm{C}$, evacuation for $30 \mathrm{~min}$, and then cooling to room temperature, the IR spectra were recorded.

\subsection{Catalytic cracking of $\boldsymbol{n}$-hexane}

$n$-Hexane cracking was performed in a continuous fixed-bed reactor. The catalyst $(1.0 \mathrm{~g})$ was loaded at the middle of a quartz reactor of length $430 \mathrm{~mm}$ and diameter 10 $\mathrm{mm}$. The operating conditions were $625^{\circ} \mathrm{C}$, atmospheric pressure, and a WHSV of $2.0 \mathrm{~h}^{-1}$. The organic components in the products were analyzed online using a gas chromatograph (SP-3420) equipped with an HP-Plot-Q capillary column and a flame ionization detector.

\section{Results and Discussion}

\subsection{Textural properties of HEU-1 zeolites after hydrochloric acid and hydrothermal treatments}

The XRD patterns of HEU-1 zeolites produced using hydrochloric acid and hydrothermal treatments are shown in Fig. 1. All the samples have the characteristic diffraction peaks of HEU-1 zeolite, indicating that the topological structure of the EU-1 zeolite remains after the hydrochloric acid and hydrothermal treatments. The relative crystallinities of the dealuminated samples are $99 \%, 95 \%, 90 \%, 87 \%$, and $92 \%$, respectively. The relative crystallinity of $\mathrm{HEU}-1(\mathrm{HCl})$ did not obviously decrease. The relative crystallinities of the samples after hydrothermal treatment decreased with increasing treatment time, in agreement with the results for $\mathrm{Y}$ and ZSM-5 zeolites after hydrothermal treatment $[18,28]$.

$\mathrm{XRF}$ analysis of the samples provides information on the total $\mathrm{SiO}_{2}$ and $\mathrm{Al}_{2} \mathrm{O}_{3}$ contents (including both framework and extra-framework species), and can be used to calculate the bulk $\mathrm{SiO}_{2} / \mathrm{Al}_{2} \mathrm{O}_{3}$ ratios. As shown in Table 1, the bulk $\mathrm{SiO}_{2} / \mathrm{Al}_{2} \mathrm{O}_{3}$ molar ratio of $\mathrm{HEU}-1(\mathrm{HCl})$ is almost identical to that of the parent sample (HEU-1). This is different from the results for ZSM-5, MOR, and $\beta$ zeolites $[19,29]$. The bulk $\mathrm{SiO}_{2} / \mathrm{Al}_{2} \mathrm{O}_{3}$ ratios of those zeolites can be adjusted by hydrochloric acid treatment. The influence of hydrochloric acid treatment on the bulk $\mathrm{SiO}_{2} / \mathrm{Al}_{2} \mathrm{O}_{3}$ ratios of the HEU-1 zeolites is negligible, which may be the result of the specific distribution of aluminum species in the HEU-1 zeolites. Peral et al. [3] reported that the framework aluminum species were anchored at the bottoms and pore mouths of 12-MR side-pockets when the EU-1 zeolite was prepared using hexamethonium ions as the structure-directing agents. Compared with zeolites such as ZSM-5 and MOR, whose framework aluminum species are randomly distributed, the framework aluminum species of HEU-1 were hardly re- moved by hydrochloric acid treatment. The bulk $\mathrm{SiO}_{2} / \mathrm{Al}_{2} \mathrm{O}_{3}$ molar ratios of the samples that underwent only hydrothermal treatment are also almost identical to that of the parent sample (HEU-1), suggesting that the bulk $\mathrm{SiO}_{2} / \mathrm{Al}_{2} \mathrm{O}_{3}$ ratio cannot be adjusted by hydrothermal treatment, unlike the results for FAU, ZSM-5, and MOR zeolites after hydrothermal treatment, whereas, the bulk $\mathrm{SiO}_{2} / \mathrm{Al}_{2} \mathrm{O}_{3}$ molar ratio of HEU-1(HT4-HCl) increased to 59.3, suggesting that the ratio could be adjusted by a combination of hydrothermal treatment and acid leaching.

The $\mathrm{N}_{2}$ adsorption-desorption isotherms of the HEU-1 zeolites after hydrochloric acid and hydrothermal treatments are shown in Fig. 2. Compared with the parent sample (HEU-1), the isotherms of HEU-1 $(\mathrm{HCl})$ have no obvious changes, but the isotherms of the samples after hydrothermal treatment display hysteresis loops at $p / p_{0}=0.4-0.9$, showing mesopore formation. Moreover, the hysteresis loop of HEU-1(HT4-HCl) at $p / p_{0}=0.4-0.9$ is more obvious than those of the samples obtained by hydrothermal treatment alone, indicating increased mesopore formation.

Table 1 gives quantitative information about the textural properties of the HEU-1 zeolites after hydrochloric acid and hydrothermal treatments. The data clearly show that compared with the parent sample (HEU-1), changes in the pore volume and specific surface area of HEU-1(HCl) can be neglected, in accordance with the XRD results. The total pore and mesopore volumes of the samples after hydrothermal treatment increase in some cases. The BET and external specific surface area are obviously enhanced, but the microporous volume and specific surface area drop slightly. This may be caused by the removal of framework aluminum species and consequent mesopore formation $[19,21,29]$. The HEU-1(HT4-HCl) and HEU-1(HT4) samples have similar pore volumes and specific surface areas.

The ${ }^{27}$ Al MAS NMR spectra of the dealuminated HEU-1 zeolites after hydrochloric acid and hydrothermal treatments are shown in Fig. 3. As can be seen, the parent HEU-1 sample shows a strong peak at $\delta=54$ from tetrahedrally coordinated framework $\mathrm{Al}$ species and a weak peak at $\delta=0$ from octahedrally coordinated extra-framework Al species [22]. After treatment with hydrochloric acid solution, the peak at $\delta$ $=0$ of HEU-1 $(\mathrm{HCl})$ almost disappeared. This suggested that the hydrochloric acid treatment can remove the extra-framework Al species of the HEU-1 zeolite but has little influence on the framework Al species. This is in accordance with the XRF results. With increasing hydrothermal treatment time, the intensity of the peak at $\delta=0$, from octahedrally coordinated extra-framework Al species, increases, and the intensity of the peak at $\delta=54$, from octahedrally coordinated framework Al species, decreases. This indicates that the framework $\mathrm{Al}$ species are removed and are converted to extra-framework Al species by hydrothermal treatment 
The XRF results show that the bulk $\mathrm{SiO}_{2} / \mathrm{Al}_{2} \mathrm{O}_{3}$ molar ratios of the samples after hydrothermal treatment are almost identical to that of the parent sample, so the extra-framework Al species still remain in the bulk HEU-1 zeolites. The peak of HEU-1 $(\mathrm{HT} 4-\mathrm{HCl})$ at $\delta=0$ almost disappears, indicating that the extra-framework $\mathrm{Al}$ species have been almost removed from the bulk, so the bulk $\mathrm{SiO}_{2} / \mathrm{Al}_{2} \mathrm{O}_{3}$ molar ratio increases from 23.1 to 59.3. Compared with the framework $\mathrm{Al}$ species, the extra-framework $\mathrm{Al}$ species are removed more easily by hydrochloric acid treatment [30]. The framework $\mathrm{SiO}_{2} / \mathrm{Al}_{2} \mathrm{O}_{3}$ molar ratios of HEU-1 zeolites can therefore be adjusted using a combination of hydrothermal and hydrochloric acid treatments.

As seen from the XRF and ${ }^{27} \mathrm{Al}$ MAS NMR results, the extra-framework Al species can be removed by hydrochloric acid treatment alone, but this is not effective in the case of the framework Al species. The HEU-1 zeolites are therefore stable in hydrochloric acid systems. The framework Al species can be removed by hydrothermal treatment and transformed to extra-framework Al species. The framework $\mathrm{SiO}_{2} / \mathrm{Al}_{2} \mathrm{O}_{3}$ molar ratios of HEU-1 zeolites can therefore be effectively enhanced using a combination of these methods.

\subsection{Acidities of HEU-1 zeolites after hydrochloric acid and hydrothermal treatments}

The distribution of acid sites was investigated using the $\mathrm{NH}_{3}$-TPD method. The $\mathrm{NH}_{3}$-TPD curves of selected samples are shown in Fig. 4. The temperature for the desorption peak maxima of the weak acid sites was set at around $200{ }^{\circ} \mathrm{C}$, and for the strong acid sites it was set at around $350{ }^{\circ} \mathrm{C}$ [31]. From Fig. 4, it is clear that the intensities and numbers of strong acid sites were almost unchanged after hydrochloric acid treatment alone [HEU-1(HCl)], and the number of weak acid sites was reduced. This is ascribed to the removal of extra-framework Al species, which is caused by hydrochloric acid treatment, and corresponds to the ${ }^{27} \mathrm{Al}$ MAS NMR results. The numbers of strong acid sites and weak acid sites are obviously reduced after hydrothermal treatment, and with increasing hydrothermal treatment time, the drop in the number of strong acid sites increases. This finding is analogous to the results obtained for ZSM-5 zeolites [16,32]. The numbers of strong and weak acid sites are obviously lower after hydrothermal and hydrochloric acid treatments. This is attributed to the conversion of framework Al species to extra-framework Al species by the hydrothermal treatment, and then removal of the extra-framework Al species by hydrochloric acid treatment. Therefore, compared with hydrochloric acid and hydrothermal treatments alone, a combination of these methods can adjust not only the weak acid sites but also the strong acid sites, so the acid strength and acid density of the HEU-1 zeolites can be modulated.
The pyridine adsorbed FT-IR spectra of dealuminated HEU-1 zeolites after hydrochloric acid and hydrothermal treatments are shown in Fig. 5. Based on previous reports on pyridine chemisorption, the bands at 1545 and $1450 \mathrm{~cm}^{-1}$ are ascribed to the adsorption of pyridine on Brönsted acid sites and Lewis acid sites, respectively; the band at $1490 \mathrm{~cm}^{-1}$ is related to a combination of Brönsted and Lewis acid sites [33]. The Brönsted and Lewis acidities were quantified from the integrated areas of the peaks at 1545 and $1450 \mathrm{~cm}^{-1}$, respectively. The integrated areas of the peaks at 1545 and $1450 \mathrm{~cm}^{-1}$ of the other samples are smaller than those of the parent sample, indicating that the HEU-1 zeolite acidity decreases as a result of hydrochloric acid and hydrothermal treatments, in agreement with the $\mathrm{NH}_{3}$-TPD results. Based on Ref. [34], the Brönsted and Lewis acidities were calculated, and are listed in Table 1. It can be seen that for the hydrochloric acid treatment [HEU-1 $(\mathrm{HCl})]$, the Brönsted acidity is slightly affected, whereas the Lewis acidity is obviously reduced from 187 to $156 \mu \mathrm{mol} / \mathrm{g}$, compared to the parent sample (HEU-1). The hydrothermal treatments cause obvious drops in the Brönsted acidities, and obvious increases in the Lewis acidities; this effect becomes more pronounced with increasing hydrothermal treatment time. Compared to HEU-1(HT4), the Brönsted acidity of HEU-1(HT4-HCl) is almost unchanged, whereas the Lewis acidity distinctly decreases from 119 to $67 \mu \mathrm{mol} / \mathrm{g}$. This result is consistent with the ${ }^{27} \mathrm{Al}$ MAS NMR results. The FT-IR results demonstrated that the Brönsted acidities are not significantly changed by hydrochloric acid treatment alone, whereas the Lewis acidities are noticeably changed; the Brönsted acidity can be modulated by hydrothermal treatment. The Brönsted and Lewis acidities of HEU-1 zeolites can therefore be effectively modulated using a combination of these methods, unlike BEA zeolites, which can be effectively modulated by acid treatment alone.

\section{$2.3 n$-Hexane catalytic cracking performance of HEU-1 zeolites after hydrochloric acid and hydrothermal treatments}

Table 2 presents the activities and selectivities of HEU-1 zeolites after hydrochloric acid and hydrothermal treatments for $n$-hexane catalytic cracking. From Table 2, it can be seen that the activities of the samples follow the order HEU-1 $>$ HEU-1 $(\mathrm{HCl})>$ HEU-1(HT2) $>$ HEU-1(HT4) HEU-1(HT4-HCl) > HEU-1(HT10) under reaction conditions of $625{ }^{\circ} \mathrm{C}$, atmospheric pressure, and a WHSV of 2.0 $\mathrm{h}^{-1}$. The order is identical to that of the acidic properties of the samples. The $n$-hexane catalytic cracking activity is proportional to the Brönsted acidities of the zeolites.

With respect to selectivity for light olefins, the selectivity for propylene is enhanced slightly by hydrochloric acid 
treatment, from $21.9 \%$ to $23.7 \%$. However, it is distinctly improved by hydrothermal treatment, from $21.9 \%$ to $31.4 \%$. This is the result of a decrease in secondary reactions, especially hydrogen transfer reactions, as a result of a reduction in the acid density. The maximum selectivity for propylene, $35.2 \%$, is obtained on HEU-1(HT4-HCl). After hydrothermal treatment, the acid densities and intensities decrease as a result of the removal of framework Al species; the hydrothermal treatment also reduces the Lewis acidities by the removal of extra-framework $\mathrm{Al}$ species, and the diffusion performance is enhanced by the removal of extra-framework Al species in the pores. Inagaki et al. [35] also found that MCM-68 zeolites dealuminated by hydrothermal treatment can enhance the selectivity for propylene in $n$-hexane catalytic cracking reactions. They emphasized that the decreased acid density and intensity were closely linked to the improvement in propylene selectivity.

The catalytic cracking of $n$-hexane involves parallel reactions [36-38]; propylene, which is the middle product, has a strong tendency to undergo hydrogen transfer reactions [39]. The propane/propylene ratio therefore represents the extent of hydrogen transfer reactions, as shown in Fig. 6. After hydrochloric acid treatment, the propane/propylene ratio is decreased slightly, remaining between 0.75 and 0.80 . HEU-1(HT2) gives the same result. After hydrothermal treatment for $4 \mathrm{~h}$, the propane/propylene ratio decreases to 0.49 . With increasing hydrothermal treatment time, the ratio is further reduced to 0.32 . The propane/propylene ratio on HEU-1 $(\mathrm{HT} 4-\mathrm{HCl})$ is 0.27 . The drops in the propane/propylene ratios indicate that the hydrogen transfer reaction is suppressed, so propylene selectivity is increased. Combined with the acidic properties, an improvement in acid density increases the extent to which the hydrogen transfer reaction occurs. The acid density is reduced by the hydrothermal and hydrochloric acid treatments, so the hydrogen transfer reaction is suppressed and propylene selectivity is enhanced.

The $n$-hexane conversion with time-on-stream was examined and the results are shown in Fig. 7. The initial conversion of $n$-hexane gradually reduces with decreasing Brönsted acidity. In the catalytic cracking of paraffins, catalyst deactivation is primarily caused by carbon deposition [40]. The catalyst lifetime can be prolonged by acidity reduction and improvement of the diffusion properties. The weaker the acidity, the higher the HEU-1 zeolite stability. Compared with the parent sample (HEU-1), the stability of HEU-1 $(\mathrm{HCl})$ is increased a little. The stabilities of the catalysts treated with steam are obviously improved; the $n$-hexane conversions on HEU-1(HT2), HEU-1(HT4), and HEU-1(HT10) drop to $60 \%$ in 18,20 , and 30 h, i.e., 10, 12 , and $22 \mathrm{~h}$ longer, respectively, compared with the HEU-1 zeolite. Up to $30 \mathrm{~h}$, the $n$-hexane conversion on HEU-1
( $\mathrm{HT} 4-\mathrm{HCl}$ ) is above $80 \%$, and up to $40 \mathrm{~h}$, the $n$-hexane conversion on HEU-1(HT4-HCl) is still above $60 \%$. Comparing HEU-1(HT4) and HEU-1(HT4-HCl), it can be seen that the stability is influenced by the Lewis acid sites, especially strong Lewis acid sites. It is difficult to desorb paraffins adsorbed on Lewis acid sites, so coke forms and causes catalyst deactivation [41]. The mesopores produced by dealumination are conducive to the diffusion of products and coke precursors, further improving the stabilities of the HEU-1 zeolites.

The changes in the selectivities for ethylene and propylene with TOS are shown in Fig. 8. For the same conversion, the ethylene selectivity on $\mathrm{HEU}-1(\mathrm{HCl})$ is slightly lower than that on HEU-1, and the propylene selectivity on HEU-1 $(\mathrm{HCl})$ is slightly higher than that on HEU-1. The ethylene and propylene selectivities on HEU-1(HT2) are little changed, similar to the case of HEU-1(HT4). On HEU-1(HT10), the ethylene selectivity obviously decreases, whereas the propylene selectivity is obviously improved. The maximum propylene selectivity and the minimum ethylene selectivity are obtained on HEU-1(HT4-HCl).

To sum up, decreasing the acidity of an HEU-1 zeolite improves light olefin selectivity and stability, but reduces the activity. It is therefore necessary to control the level of dealumination. Generally, treatment of the zeolite at $600{ }^{\circ} \mathrm{C}$ for $4 \mathrm{~h}$ gives the best results. The acidic properties of EU-1 zeolites can be adjusted effectively using a combination of hydrothermal and hydrochloric acid treatments, in order to further enhance propylene selectivity in $n$-hexane catalytic cracking.

\section{Conclusions}

The textural properties of HEU-1 zeolites after hydrochloric acid treatment, hydrothermal treatment, and a combination of these treatments were investigated. As can be seen from the results, hydrochloric acid treatment alone can only remove the extra-framework species and adjust the weak acid sites; hydrothermal treatment alone can remove the framework species and adjust the strong acid sites. Hydrothermal treatment and then hydrochloric acid treatment can adjust the strong acid sites and the weak acid sites, and form mesopores. In $n$-hexane catalytic cracking reactions, the hydrogen transfer reaction was significantly suppressed on HEU-1(HT4-HCl) and the maximum propylene yield and selectivity were obtained; these were $7.8 \%$ and $13.3 \%$ higher, respectively, than those on the parent sample. The stability was also noticeably enhanced.

Full-text paper available online at Elseiver ScienceDirect http://www.sciencedirect.com/science/journal/18722067 